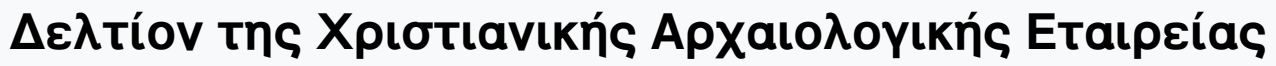

Tóp. 4 (1966)

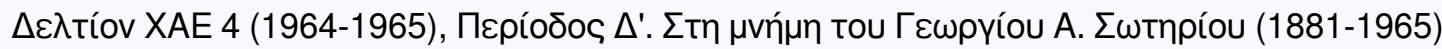

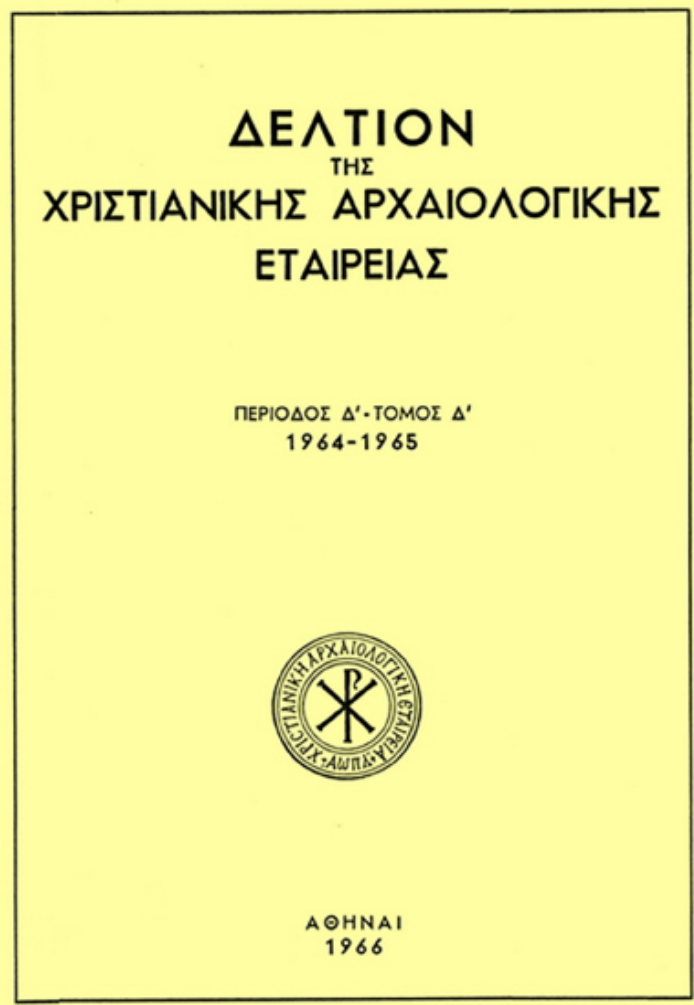

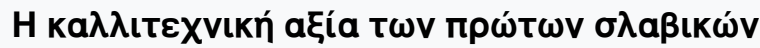

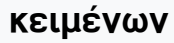

Ant. DOSTÁL

doi: $\underline{10.12681 / \text { dchae. } 770}$ 


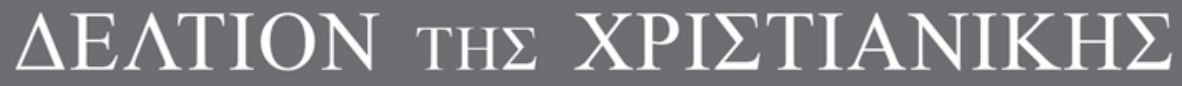 APXAIO $\Lambda$ OГIKH $\Sigma$ ETAIPEIA $\Sigma$}

La valeur artistique des premiers textes slaves

Ant. DOSTÁL

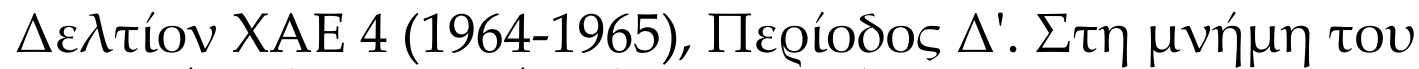

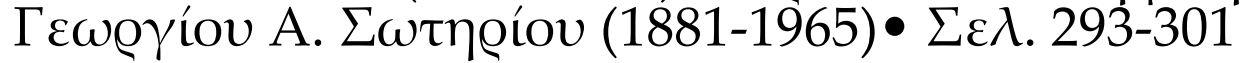

A@HNA 1966 


\section{LA VALEUR ARTISTIQUE DES PREMIERS TEXTES SLAVES}

1. La seconde moitié du IXe siècle est l'époque de la création de la culture slave écrite qui prit son origine à l'occasion de l'arrivée de la mission byzantine en Grande Moravie avec Constantin le Philosophe et son frère Méthode à la tête. Les Slaves n'avaient pas, avant ce temps, leur propre écriture. Selon Chrabr qui décrit dans son traité intitulé $O$ pismenechъ (Des Lettres) de l'invention de l'écriture slave, les Slaves se servaient de simples signes, s'ils avaient besoin de se rappeler quelque chose. Constantin inventa l'écriture slave, traduisit les premiers textes et, avec son frère Méthode et leurs disciples, posa les premiers fondements de la littérature slave et ainsi de la culture slave écrite en général. Les Évangiles furent traduits les premiers, à savoir le recueil de péricopes des Évangiles, appelé Évangéliaire. Plus tard les Évangéliaires furent remaniés et augmentés par l'interpolation des passages manquants de sorte que tout le texte des Évangiles fut traduit en slave (en vieux slave) encore pendant la vie de Constantin.

Plusieurs chercheurs, slavisants ou autres, se sont occupés de l'analyse de ces premiers textes. Ils sont toujours parvenus à constater une grande valeur artistique de ces premiers textes. Ces traductions ont été souvent comparées aux autres traductions de l'Évangile (p.ex. à la traduction gothique), bien que cette comparaison ne fût pas toujours tout à fait correcte, parce que ces différents textes avaient pris leur origine dans les conditions très variées. Très souvent les chercheurs se sont posé la question, comment est-il possible que les premiers textes slaves qui jamais fussent écrits, se distinguent d'une si haute valeur artistique. On attend a priori que les textes qui sont créés au début même de la littérature, les premiers textes écrits dans une langue littéraire nouvelle, sont très simples, sans valeur, sans une souplesse stylistique parce que la tradition de la langue littéraire manque complètement. Très souvent il dure très longtemps qu'on parvient au moment de pouvoir créer des textes de valeur dans une langue littéraire nouvelle. Comment s'expliquer la situation des premiers textes slaves? Dans le cas des premiers 
textes slaves, ce sont des textes par lesquels la littérature slave commence sans avoir une tradition littéraire ${ }^{1 .}$

On s'expliquait ce fait de différentes manières. On supposait d'abord la possibilité que les Slaves auraient pu avoir une «ittérature»avant l'arrivée de Constantin et Méthode en Grande Moravie. Si l'on considère tous les faits historiques que nous avons à la disposition, il est impossible d'accepter cette hypothèse. Dans son traité cité plus haut, Chrabr indique une date de l'invention de l'écriture slave. Si l'on la déchiffre selon l'ère byzantine, on reçoit l'an 855 , c.-à-d. suivant cette indication de la date de l'invention de l'écriture slave, on pourrait supposer l'existence d'une littérature slave avant la mission byzantine en Grande Moravie. Mais on sait très bien toutes les difficultés de cette date de Chrabr (on accepte le plus souvent que l'indication de Chrabr n'est pas faite suivant l'ère byzantine et de plus, on connaît la contradiction des témoignages historiques, si l'on acceptait cette hypothèse). Néanmoins quelques slavisants, surtout bulgares, acceptent cette hypothèse qui n'est pas acceptée par d'autres chercheurs. - Une autre explication s'appuie sur le témoignage dans la réponse de Michel III à Constantin (cf. la Vie de Constantin, chap. XIV) que "tous les habitants de Thessalonique parlent purement slave». On en déduit que les Slaves vivant en Byzance, à Thessalonique et dans ses environs ou autre part dispercés sur le territoire de Byzance, étaient bilingues; qu'ils étaient capables de parler le slave ainsi bien que le grec. On déduit de ce témoignage que pour les Slaves en Byzance, il fallut traduire la Bible avant la mission byzantine en Grande Moravie, de sorte que des textes slaves existaient avant cette mission. Cette hypothèse a ses grandes difficultés à son tour. Si les Slaves vivant en Byzance étaient bilingues, à quoi bon de leur traduire la Bible en slave? Aucun manuscrit, aucun témoignage ne s'est conservé en faveur de cette hypothèse. - On rappelle enfin, à juste titre, le grand génie et talent de Constantin. Constantin connaissait sûrement plusieurs langues, il fut très érudit sur la culture byzantine-grecque. Il construisit le système graphique pour les Slaves très parfait et précis - l'alphabet slave; la glagolite est tellement précise qu'elle témoigne en faveur des grandes connaissances philologiques et linguistiques de Constantin. Comme on peut supposer très réellement que Constantin connut également bien le slave que le grec, on a déduit de ce fait que Constantin fut

1. M. W e i ng a r t, Rukověţ’jazyka staroslověnského, Prague 1937; K. H o r á l e k, Evangeliare a čtveroevangelia, Prague 1953: A. D o s t ál, Clozianus, Prague, 1954. 
d'origine slave ou au moins du côté de sa mère parce qu'il connut cette langue comme une langue maternelle. On ne peut pas prouver cette hypothèse, parce qu'on n'a point de témoignages directes. Mais pour notre étude, ce qui est d'une grande importance, c'est la supposition réelle du grand génie de Constantin. Un tel traducteur, écrivain byzantin, membre de plusieurs délégations diplomatiques de Byzance, un connaisseur parfait de la Bible et d'autres textes, tout cela nous démontre clairement que Constantin fut un homme de génie et un homme de culture tout à fait extraordinaire. Il est incontestable que ces qualités de Constantin jouèrent un grand rôle dans sa mission en Grande Moravie. Ce qui nous intéresse ici, e'est la question en quoi consiste la valeur artistique, sans aucun doute très grande, des premiers textes slaves créés par Constantin. Il faut se rappeler quelques faits d'ordre philologique, mais avant tout, il faut prendre en considération aussi la méthode de l'appréciation de la qualité des textes littéraires anciens.

2. La question de la critique des textes vieux du point de vue de l'art littéraire, est très compliquée et très difficile. Il est compréhensible que l'existence d'un texte à l'époque dans laquelle il n'y a rien d'autre, constitue quelque chose d'extraordinaire. De ce point de vue, les premiers textes pourraient constituer un grand témoignage culturel, historique, etc. Le même texte existant dans une époque dans laquelle il y a une abondance de textes de toute sorte serait, peut - être, sans valeur. Il faut dire avec accent que ceci n'est point le cas des premiers textes slaves. Il s'agit vraiment de textes d'une grande valeur artistique.

La valeur de ces textes peut et doit être constatée - selon mon avis - seulement en prenant en considération le contexte de l'époque de la naissance de ces textes. Toute époque et tout degré de l'évolution sociale a de différentes exigences et différents critéria pour l'art littéraire de même que pour tout autre sorte de la création artistique. On peut objecter à ce point de vue qu'une vraie œuvre artistique ne peut pas respecter les modes ou les exigences de la société, mais très souvent l'art est étroitement lié au peuple, c.-à-d. au peuple déterminé historiquement. De l'autre côté, on doit avouer qu'il existe des œuvres artistiques dont la valeur surpasse l'évolution du temps, mais on doit ajouter que c'est surtout le cas des textes qui ne s'adresse pas à un peuple concret mais à l'homme en général. Si nous pensions aux traductions, ce fait de l'œuvre qui surpasse le temps, se rattache à l'original mais non aux traductions. Chaque époque se choisit et se traduit des textes dont elle sent le besoin. Quelques textes ont été traduits plusieurs fois sans prétendre une ambition 
de faire la traduction définitive et dernière. Les autres textes sont traduits par une époque et sont complètement négligés par l'autre parce que l'époque ne sent pas besoin d'avoir ces traductions.

En quoi constitue la valeur artistique d'un texte comme de l'œuvre artistique?

Avant de répondre à cette question, il faut prendre en considération les qualités de la matière contenue dans le texte ainsi que la langue dans laquelle le texte est écrit.

Les textes dont nous nous occupons dans cet article, ont quelques traits extraordinaires et singuliers. Il s'agit de textes très engageants du point de vue de leur contenu. Ce caractère de ces textes fut très sensible aussi dans l'époque de Constantin et Méthode, parce qu'il était en relation directe avec le but de leur mission. Les deux frères furent appelés par Rostislav et délégués par Michel III pour y divulguer le christianisme.

On peut se poser la question, est-ce qu'on peut changer le contenu de ces textes, c.-à-d. est-ce que le contenu de ces textes pouvait être influencé par la volonté du traducteur. Aucun texte n'est absolument stable, au contraire, le texte vit comme des êtres vivants: il parle aux gens de leur temps, il est reçu ou rejetté, changé, «expliqué» plusieurs fois; quelque fois il est changé avec une intention spéciale (pour "corriger» le vrai sens de ces textes, pour les "améliorer», etc.). Ce sont les occasions, très souvent nombreuses d'ailleurs dans la vie des textes, pendant lesquelles on peut remanier le texte, changer légèrement ou essentiellement le contenu du texte. La quantitié de ces changements est en relation directe très souvent avec le type du texte. Pour les Évangiles, on peut dire que même ces textes étaient très souvent exposés aux changements du point de vue du contenu. On sait très bien qu'on a plusieurs rédactions de ces textes et un nombre très grand des textes légèrement changés dans le cadre de ces rédactions.

Les changements dans le texte de l'Évangile étaient pour la plupart légers, à côté des changements essentiels qui changeaient le sens profondément ce qui, quant aux Évangiles, avaient de grandes et sérieuses conséquences dans l'histoire de l'Église.

Les quatre Évangiles peuvent servir d'exemple pour tels remaniements et changements du texte. Il y a quatre Évangiles, chacun d'eux raconte la même histoire - la vie de Jésus. La coïncidence entre ces textes est plus ou moins grande. En somme, ils nous racontent le même contenu, jamais ils ne sont en contradiction l'un à l'autre, mais ils se complètent mutuellement. Ce qui est dans un Évangile, peut être com- 
plété par des détails contenus dans l'autre Évangile et vice versa. Et malgré cela, il n'y a pas de coinncidence complète et parfaite. Regardons p.ex. le début du récit de différents Évangiles. Les trois premiers (ceux de St. Mathieu, St. Marc et St. Luc) commencent leur récit généalogiquement: ils racontent l'origine de la famille dans laquelle Jésus naquit. Ensuite c'est l'histoire de la vie de Jésus, interpolée par des épisodes de la vie terrestre de Jésus jusqu'à sa mort et aux évènements après sa mort. Le quatrième Évangile, celui de St. Jean, commence par une réflexion philosophique sur le Logos, mais plus tard il quitte cette voie de réflexion et raconte de la vie de Jésus, de la manière égale aux trois Évangiles premiers. Du point de vue de la langue de ces Évangiles, il y a aussi de grandes différences ${ }^{1}$.

Du point de vue du genre littéraire, les textes des Évangiles sont des récits épiques, avec une grande quantité d'épisodes qui sont très en faveur chez les auteurs des Évangiles. Ces épisodes sont quelques fois assez indépendants, ils pourraient facilement être racontés ou lus séparément à une fête ou à un jour fixe de l'année ecclésiastique. Chaque épisode contient toujours une maxime morale ou une pensée théologique ou philosophique. Ces épisodes contiennent très souvent des paroles directes de Jésus ou des personnes y engagées. Du point de vue de la propagande du christianisme, ce sont d'excellents textes, choisis et mis en contexte d'une maîtrise exceptionnelle, parce qu'ils sont destinés à l'auditeur ou au lecteur très simple, évitant chaque haute abstraction philosophique et sont capables d'expliquer les idées les plus compliquées et les plus abstraites d'une manière facile et légère. De l'autre côté, il ne faut pas oublier que même cette forme du récit ne peut pas priver l'auteur du texte du devoir de se servir, çà et là, relativement assez souvent, d'une terminologie philosophique. Il y a là alors une terminologie semblable aux autres genres de textes scientifiques, de textes visant à un sujet.

Cette conception fondamentale des textes des Évangiles ne peut être rompue en aucun cas. A côté des exigences dont nous venons de parler, il y a encore un autre fait, très important, c.-à-d. le contenu concetré autour du Christ, par la situation centrale de lui dans le chritianisme ce qui pose à l'auteur plusieurs exigences. Le sujet très simple, des épisodes - histoires prises de la vie de tous les jours, tout cela pouvait être formulé par des mots simples qui ne visent pas la beauté de la formulation et même ayant la possibilité d'être très proche au peuple

1. H. P e r n o t, La langue des Évangiles, Paris 1927. 
simple, à la manière de s'exprimer, connue chez les gens peu érudits, simples, même vulgaires, etc.

Il est très intéressant de rappeler un fait important. Le texte des Évangiles et de la Bible en général, fut traduit en centaines de langues. A priori, on pourrait attendre à côté des formes très sublimes de ces textes aussi des formations très banales dans les traductions. Mais il est très intéressant que nulle part ces textes n'ont pris une forme vraiment banale. La matière sublime de ces textes exigeait toujours aussi une forme sublime. On tâchait d'atteindre très souvent une formation élégante, belle, précise, «divine» comme l'était aussi le contenu de ces textes. Jamais cette matière ne menait à une formulation banale, grossière, bref une formulation qui pourrait abbaisser la sublimité de ces textes. Il y avait aussi des essais de rapprocher ces textes à la langue très moderne, courante, parlée, mais on a toujours aboutit à faire une traduction extraordinairement soutenue. Nulle part cette matière n'est devenue banale et grossière. On voit très bien comment la matière d'un texte exige une certaine conception, une certaine formulation et un certain choix de procédés linguistiques pour exprimer ces textes. On sent très bien ces faits aussi dans les premières traductions des textes de l'Évangile en slave.

Quant à la formulation et la stylisation du contenu de ces textes, il y a des choses suivantes qui doivent être prises en considération. Chaque sujet parlant regarde, touche, etc. bref conçoit des évènements réels d'une certaine manière qui est, très souvent, propre et caractéristique au sujet parlant. S'il veut communiquer celui qui parle ce qu'il a conçu à un autre sujet parlant auquel il s'adresse, il doit le styliser, c.-à-d. le dire d'une certaine manière et la formulation linguistique, c.-à-d. le choix de procédés du système linguistique dans lequel il communique ses idées à l'auditeur, reflète sa conception de l'évènement réel. Le choix des mots n'est pas sans importance, ainsi que la construction syntactique choisie, etc., parce que le même évènement peut être exprimé et formulé d'une manière très différente. Le sujet parlant veut exprimer sa formulation également comme il l'a conçu. La matière sublime des Évangiles influence probablement le choix des mots par l'auteur, l'emploi des locutions populaires ou érudites, le choix des mots abstraits ou concrets, etc. L'impression finale évoquée par l'auteur de cette formulation linguistique chez l'auditeur ou chez le lecteur est en relation directe avec le but de ce texte. Une bonne traduction, traduction de valeur, c.-à-d. artistique, devient ensuite une partie de la richesse culturelle du peuple, de la société qui l'accepte. S'il s'agit du texte utile à l'explication des faits quotidiens, 
de tous les jours, des joies, des soucis, etc., comme c'est le cas des Évangiles, un tel texte appartient vraiment à la richesse culturelle de la nation parlant dans cette langue.

L'art de l'auteur, son génie suivant lequel l'auteur d'une telle traduction savait répondre à toutes les exigences de ce texte, la compréhension du texte de l'original et l'expression linguistique, tout cela sont des agents très importants qui jouent un grand rôle dans l'appréciation critique de la valeur artistique d'un texte littéraire.

On peut parler aussi de l'esthétique d'une traduction, mais je pense que cela résulte déjà de ce qu'il a été dit plus haut.

3. Les questions linguistiques, les éléments linguistiques sont très importants pour l'évaluation du texte du point de vue de sa valeur artistique. La langue, c.-à-d. le système linguistique constitue pour l'artiste littéraire une sorte de matière première dont l'œuvre littéraire est faite. D'abord, il est très important pour l'artiste littéraire que la langue soit si développée, si riche et si convenable à exprimer toutes les nuances des pensées, comme le sujet, la matière du texte l'exige. Nous avons des exemples très nombreux de la disharmonie entre le sujet du texte, le but de l'artiste littéraire et le degré de l'évolution de la langue. Si p.ex. la Iangue littéraire sortit de son épanouissement, si elle était restreinte, il arrive qu'elle ne suffit pas aux exigences de l'auteur et de la matière qui doit être formulée par des éléments linguistiques. Très souvent on voit que le développement de la littérature va en coïncidence avec le développement de la langue. Et qu'est-ce que le développement de la langue? Il ne s'agit pas seulement de l'enrichissement du lexique, mais souvent encore d'autres changements dans le système de communication. On voit p.ex. que le rythme des changements de la langue est très souvent en relation avec le développement littéraire et culturel. La langue se simplifie ou se complique suivant les besoins de ceux qui la parlent et écrivent: les changements phonologiques se cristalisent, la morphologie devient plus souple en accentuant des formes productives et en écartant les formes anomales, la syntaxe souligne certaines constructions des phrases, le lexique s'enrichit, etc. On peut comparer ces qualités de la langue littéraire au degré de la technique de peinture, etc.

La langue littéraire n'a pas, à chaque époque, les mêmes fonctions. Elle peut avoir la fonction poétique p.ex. à la fois qu'elle n'est pas la langue de l'administration ou de l'armée, de la science, etc. Les fonctions d'une langue littéraire sont en relation directe les unes aux autres. $\mathrm{Si}$ une langue littéraire est repoussée de l'emploi en plusieurs fonctions, 
elle ne peut, très souvent, satisfaire ni la fonction qui lui reste. Une langue littéraire qui n'est pas développée peut freiner l'artiste littéraire dans son travail.

Le manque d'une tradition de la langue littéraire peut être un grand obstacle dans l'emploi de cette langue pour une œuvre artistique littéraire. Il arrive que les textes rédigés dans une telle époque ne peuvent pas avoir le niveau artistique à cause de la langue, ils sont primitifs, etc.

4. La valeur artistique des premiers textes slaves, des traductions des Évangiles, est très haute. Le traducteur dut être un homme de génie, de talent, un artiste littéraire véritable, un styliste virtuose sans aucun doute. Constantin eut une érudition très haute, il dut avoir des connaissances extraordinaires, une capacité admirable de formuler et de styliser le texte. Sans cela on ne pourrait pas se figurer l'existence d'une traduction qu'il nous a laissée. Les connaissances des langues chez Constantin furent admirables. Il connut le grec et le slave également très précisément. Il inventa d'abord un alphabet très précis pour pouvoir écrire bien la langue slave avec tous ses sons mouillés et avec toutes les différences phonologiques du grec byzantin. Il adapta même un peu la langue slave qu'il parlait: c'est une langue très régulière, logique, pratique et précise. Dans la morphologie du vieux-slave, Constantin fit ressortir des types productifs, il souligna à la fois le caractère logique des formes morfologiques $d u$ vieux-slave. Dans la richesse du lexique cette langue est sans bornes: d'un côté Constantin développe des règles de la formation des mots d'origine slave, de l'autre côté il introduisit en vieux-slave encore des principes grecs (p.ex. l'emploi des mots composés, des calques). Et de plus, Constantin se servit d'un certain nombre de mots grecs adaptés seulement sans traductions dans le texte slave (c'étaient probablement les mots qui étaient courants dans le langage des Slaves en Byzance). Quant à la syntaxe, il se servit très souvent des modèles de construction de la syntaxe du grec en gardant les principes slaves originaux. Il faut rappeler que c'est surtout la syntaxe du grec du Nouveau Testament, courant en Byzance, qui peut être reconnue dans la syntaxe des premiers textes vieux-slaves.

Constantin unifia la langue de tous les quatre Évangiles (de ce point de vue il y a des différences dans le texte grec). Dans la traduction de Constantin, on voit très souvent une conception légèrement différente de l'original grec. Constantin formula souvent sa stylisation d'une manière rappelant le langage parlé, avec des métaphores simples, légères et aimables en conservant relativement très fidèlement le texte grec. 
Les locutions dans la langue de ses traductions rappellent quelque fois l'imagination simple, presque d'une naïvité enfantine aimable et convainquante. Sans aucun doute Constantin fut maître et un grand artiste littéraire. C'était un artiste qui sut non seulement soumettre la langue à ses exigences mais qui sut, de l'autre côté, la stabiliser et rendre capable d'exprimer les nuances les plus fines de la pensée. Si l'on prend en considération qu'il s'agit là d'une langue sans tradition littéraire, l'importance du génie de Constantin ressort encore de plus. C'était un grand bonheur pour les Slaves que c'étaient Constantin et Méthode, hommes de telles qualités, qui furent choisis pour la mission en Grande Moravie. Ce choix fut décisif pour les débuts de la culture slave écrite. Les deux frères fondèrent de cette manière la littérature slave, la tradition littéraire chez les Slaves, ils leur donnèrent un instrument culturel de tout premier ordre, instrument qui permit aux Slaves de s'approprier toutes les richesses de la culture écrite des Byzantins et par leur intermédiaire, de la culture antique, orientale et, de l'autre côté, de la culture européenne occidentale. Les Slaves se servaient de cette langue littéraire comme d'une langue interslave pendant mille ans, bien que la littérature écrite dans les langue nationales, à côté du slavon, se développât bientôt parallèlement.

ANT. DOSTÁL 